\title{
Acute aphasia after right hemisphere stroke
}

\author{
Géraldine Maillard Dewarrat · Jean-Marie Annoni · \\ Eleonora Fornari · Antonio Carota • \\ Julien Bogousslavsky $\cdot$ Philippe Maeder
}

Received: 17 August 2008/Revised: 1 February 2009/Accepted: 26 February 2009/Published online: 12 April 2009

(C) Springer-Verlag 2009

\begin{abstract}
Right hemispheric stroke aphasia (RHSA) rarely occurs in right- or left-handed patients with their language representation in right hemisphere (RH). For right-handers, the term crossed aphasia is used. Single cases, multiple cases reports, and reviews suggest more variable anatomo-clinical correlations. We included retrospectively from our stroke data bank 16 patients (right- and left-handed, and ambidextrous) with aphasia after a single first-ever ischemic RH stroke. A control group was composed of 25 successive patients with left hemispheric stroke and aphasia (LHSA). For each patient, we analyzed four modalities of language (spontaneous fluency, naming, repetition, and comprehension) and recorded eventual impairment: (1) on admission (hyperacute) and (2) between day 3 and 14 (acute). Lesion volume and location as measured on computed tomography (CT) and magnetic resonance imaging (MRI) were transformed into Talairach stereotaxic space. Nonparametric statistics were used to compare impaired/nonimpaired patients. Comprehension and repetition were less frequently impaired after RHSA (respectively, 56\% and 50\%) than after LHSA (respectively, $84 \%$ and $80 \%, P=0.05$ and 0.04 ) only at hyperacute phase.
\end{abstract}

G. M. Dewarrat $(\bowtie) \cdot$ J.-M. Annoni · A. Carota

Department of Neurology, Centre Hospitalier Universitaire

Vaudois (CHUV) and University of Lausanne,

Rue du Bugnon, 46, 1011 Lausanne, Switzerland

e-mail: geraldine.maillard-dewarrat@chuv.ch

E. Fornari · P. Maeder

Department of Radiology, Centre Hospitalier Universitaire

Vaudois (CHUV) and University of Lausanne,

Lausanne, Switzerland

J. Bogousslavsky

Genolier Swiss Medical Network, Clinique Valmont-Genolier,

Glion-sur-Montreux, Switzerland
Among RHSA, fewer left-handers/ambidextrous than righthanders had comprehension disorders at second evaluation $(P=0.013)$. Mean infarct size was similar in RHSA and LHSA with less posterior RHSA lesions (caudal to the posterior commissure). Comprehension and repetition impairments were more often associated with anterior lesions in RHSA (Fisher's exact test, $P<0.05$ ). Despite the small size of the cohort, our findings suggest increased atypical anatomo-functional correlations of RH language representation, particularly in non-right-handed patients.

Keywords Aphasia - Cerebrovascular disease . MRI

\section{Introduction}

In the stroke literature, the prevalence of crossed aphasia in right-handed patients with neither family history of lefthandedness nor previous history of brain disease is $0.4-3.5 \%$ of all aphasic syndromes $[1,5,10,13]$. The lesion distribution and the recovery pattern have been reported to be similar those of uncrossed language disorders [8, 13]. However, atypical aphasia anatomo-functional correlations (i.e., aphasic syndrome which does not fit with the specific ischemia site, for example, conduction aphasia in extrainsular strokes) have been found to be more common in right hemispheric stroke aphasia (RHSA) (35\% after right hemisphere (RH) lesions [2] versus $13 \%$ after LH lesions [3]). Both oral and written modes of language comprehension are described as seldom impaired after RHSA, and written language as more affected than oral speech [12]. However, crossed aphasia does not account for all RH lesion inducing language disturbances. The study of left-handed and ambidextrous patients, in whom both the LH and RH are supposed 
to be involved in language, takes into account the impact of handedness on language organization in such patients [14, 17]. It is also important to differentiate the hyperacute/acute from more chronic phases, since damage to a functional network often produces reorganizations $[6,25,26]$. The aim of our study was to retrospectively collect aphasiological, handedness, and anatomical characteristics of an unselected group of RHSA patients [16], during hyperacute (i.e., on admission) and acute (first 2 weeks after stroke) phases compared with a control group of LHSA patients [25]. We could characterize RHSA eventual atypical clinical and radiological features, even when ambidextrous/left-handed (non-right-handed) patients were considered.

\section{Methods}

\section{Patient inclusion}

We based our study on a cohort of patients from the Lausanne Stroke Registry (LSR) and the Geneva Neurological Wards Data Banks from January 1990 to December 2002. We looked at the clinical and radiological data reported in the charts of all patients with language impairment after RHS. Among these patients, we selected the patients with the following inclusion criteria:

1. A single, first-ever ischemic RH stroke with a single lesion on a brain CT or MRI performed during the first 2 weeks after the event.

2. Aphasia attested by a neurologist in the emergency ward. Our criteria to retain aphasia were: presence of a significant anomia (difficulty in naming simple objects such as watch, pen, glasses, button) confirmed by clinical evaluation of naming, plus one of the following observations: (1) presence of paraphasic errors in naming or repetition, (2) comprehension impairment, (3) transformation in reading (paralexia) or (4) in writing (paragraphia), and (5) absence of confusional state. In order for the patients to be included, aphasiological data present in the medical chart had to contain a semiquantitative or qualitative description of language behavior in a few basic tasks (spontaneous language fluency, comprehension, repetition, naming), as well as a description of eventual paraphasia, paralexia, and paragraphia.

3. The presence, in the medical chart, of satisfactory description of two language evaluations: the first on admission in the emergency ward and the second more extensive between the 3rd and 14th days, performed by a speech therapist.

The same inclusion criteria were applied to a cohort of consecutive patients with LHSA enrolled in the LSR in
2002. Of an initial cohort of 25 RHSA and 36 LHSA, 9 RHSA patients and 11 LHSA patients were excluded for one of the following reasons: (1) complete recovery in the first $24 \mathrm{~h}$ after stroke (TIA) (2 RHSA/4 LHSA); (2) previous cognitive impairment, drowsiness, somnolence or treatment with psychotropic drugs (3 RHSA/3 LHSA), (3) epilepsy and medical complications (4 RHSA/4 LHSA). Other causes of language expressive disorders such as inattention or anxiety had to be excluded. We classified handedness (righthanded, ambidextrous, or left-handed) with the help of a semistructured interview relying on common tasks [preferred hand for writing, drawing, throwing, striking match, opening box (lid) and handling scissors, toothbrush, knife, spoon, broom (upper hand)] [24].

Evaluation of aphasia

Both evaluations were conducted by two authors (J.-M.A. and G.M.D.) from the clinical charts.

For the first evaluation, in the $48 \mathrm{~h}$ post stroke onset (hyperacute phase), the emergency ward clinical records were extracted from the charts. Language picture was estimated clinically from the quantitative and qualitative information on language behavior in the few mentioned basic tasks (spontaneous language fluency, comprehension, repetition, and naming).

For the second assessment, during the acute phase (days $3-14$, mean $8 \pm 2.5$ days), we collected, from the same patients, the data of the complete language examination done by a speech therapist, using tests belonging to standardized batteries such as the French version of the Boston Diagnostic Aphasia Examination (BDAE) [21] and/or the modified version of the Protocole Montréal-Toulouse d'examen de l'aphasie [23].

To facilitate hyperacute and acute comparison of language impairment, in a heterogeneous population, and to perform an anatomo-clinical association, we applied a simple two-level aphasia score (normal $=0$, clinically impaired or outside normal values $=1$ ) to the data recorded during the hyperacute and acute phases concerning the four following modalities: fluency of spontaneous discourse, naming, repetition, and comprehension.

Moreover, we collected the results of neurological and neuropsychological examinations performed during the acute phase.

Imaging techniques and anatomical evaluation

The imaging study was based on either CT or MRI. Eleven patients in the RH group and all patients [25] in the LH group underwent transverse 5-mm thick fast spin-echo T2-weighted MRI (TR, 3,800 ms; TE, $90 \mathrm{~ms}$; NEX, 2; FOV, $173 \times 230$; matrix, $190 \times 256$; $30 \%$ gap) performed 
between the 2 nd and the 10th day. Five patients in the RH lesion group had only a brain CT done during the first 3 days. This was performed with $5-10 \mathrm{~mm}$ slices in the orbitomeatal plane, from the foramen magnum to the vertex with contrast injection. The precise anatomical evaluation was obtained by using the normalized coordinate system of Talairach and Tournoux [27]. MR T2 or CT volumes were resliced, visually inspected in a $3 \mathrm{D}$ view, and rendered into the Talairach stereotaxic space using dedicated software (Brain Voyager 2000, V 4.9.6.0, Brain Innovation BV, Maastricht, The Netherlands). Two of the authors (P.M. and G.M.D.) analyzed each lesion separately, and then found agreement on the implicated Talairach grid cuboids.

We individually associated aphasic impairments in comprehension, repetition, and spontaneous language fluency with the implicated Talairach cuboids. For each of these language modalities, we selected cuboids in which a lesion was present in at least $50 \%$ of the impaired patients, so we could confront ( $\chi^{2}$ on contingency tables) injured brain areas with specific language impairment.

\section{Statistics}

The two main statistical analyses were carried out on raw data with SPSS version 10 for Windows software. Due to the small sample, we used nonparametric tests for the statistical assessment. The first analysis tested the influence of left versus right hemisphere on severity of impairment for each modality of language at two different times (hyperacute and acute phases). This comprised a group comparison test between RH and LH lesions, respectively, during the hyperacute and acute phases, with the mean of $\chi^{2}$ test. Second, we performed a correlation analysis using contingency tables. For each Talairach cuboid and for each language modality, we constructed a $2 \times 2$ contingency table to determine whether the status of the cuboid (i.e., normal/injured) was associated with impairment (i.e., present/not present). Pearson $\chi^{2}$ and Fisher's exact tests were used on the raw data to determine whether the location of the lesions could affect impairment, depending on the modality of language and the hemisphere. For all these tests, and due to the small number of patients, a $P$ value of 0.05 was considered to be significant.

\section{Results}

Demographic and neurological data

Sixteen RHSA patients [13 men (i.e., 82\%); 7 right-handers, 3 ambidextrous, 6 left-handers] with mean age $61.7 \pm 13.5$ years (range $44-87$ years) were included, and 25 LHSA patients [14 men (i.e., 56\%)] with mean age
Table 1 Demographic and clinical features of patients with RHSA and LHSA

\begin{tabular}{llll}
\hline Characteristics & RH & LH & $p$ \\
& $n=16$ & $n=25$ & \\
\hline Age, mean \pm SD, years & $61.7 \pm 13.5$ & $63.9 \pm 11.5$ & 0.57 \\
Age for fluent aphasia, years & $61.8 \pm 9.9$ & $66.1 \pm 13.4$ & 0.49 \\
Age for nonfluent aphasia, years & $61.6 \pm 13$ & $62.8 \pm 13.8$ & 0.8 \\
Sex ratio, men, \% & $82(n=13)$ & $56(n=14)$ & 0.09 \\
Handedness, \% & & & \\
Right-handed & $44(n=7)$ & 100 & 0.001 \\
Left-handed & $37(n=6)$ & 0 & 0.001 \\
Ambidextrous & $19(n=3)$ & 0 & 0.001 \\
Neurological features & & & \\
SMH, \% & $44(n=7)$ & $44(n=11)$ & 0.98 \\
MH, \% & $56(n=9)$ & $28(n=7)$ & 0.07 \\
Hemianopsia, \% & $38(n=6)$ & $56(n=14)$ & 0.24 \\
Spatial neglect, \% & $44(n=7)$ & $16(n=4)$ & 0.05 \\
Anosognosia, \% & $13(n=2)$ & $20(n=5)$ & 0.5 \\
\hline
\end{tabular}

$R H$ right hemisphere, $L H$ left hemisphere, $S M H$ sensorimotor hemisyndrome, $M H$ motor hemisyndrome

$63.9 \pm 11.5$ years (range 38-89 years) were included in the control group (Table 1).

Mean age and gender did not significantly differ between the two groups, while handedness was not similarly distributed. The LHSA group included only righthanded patients, whereas the RHSA group included lefthanders $(n=6 ; 37 \%)$ and ambidextrous $(n=3 ; 19 \%)$.

Spatial neglect was significantly more present after RHSA (44\%), see Table 1, while motor/sensory deficits, hemianopsia, and anosognosia did not differ.

Language in the hyperacute and acute phases

Distribution of language disorder in the hyperacute and acute phases is presented in Table 2.

We compared also the language behavior between rightand non-right-handers after RHSA. There was no difference between both subgroups in the hyperacute phase for all modalities of language.

In RHSA, non-right-handers had a better outcome in comprehension, with only $11 \%$ (1 patient) still having comprehension impairment at the second evaluation, compared with $42 \%$ of right-handers $\left(\chi^{2}=6.11, P=0.013\right)$. We did not find the same significant difference for repetition $\left(\chi^{2}=0.42, P=0.3\right)$, for spontaneous language fluency $(\chi=1.17, P=0.28)$ or for naming $(\chi=0.78, P=0.4)$.

Imaging findings

Lesion load as measured by the mean numbers of implicated Talairach cuboids did not differ between groups 
Table 2 Language disorder in the hyperacute and acute phases

\begin{tabular}{|c|c|c|c|c|}
\hline Language modality & $\mathrm{RH}(n=16)$ & LH $(n=25)$ & $\chi^{2}$ & $P$ \\
\hline \multicolumn{5}{|l|}{ Hyperacute phase } \\
\hline Comprehension, $\%,(n)$ & $56(n=9)$ & $84(n=21)$ & 3.8 & 0.05 \\
\hline Repetition, \% ( $n$ ) & $50(n=8)$ & $80(n=20)$ & 4.1 & 0.04 \\
\hline Spontaneous language fluency, $\%(n)$ & $56(n=9)$ & $68(n=17)$ & 0.58 & 0.44 \\
\hline Naming, \% (n) & $100(n=16)$ & $100(n=25)$ & 0 & 1 \\
\hline \multicolumn{5}{|l|}{ Acute phase } \\
\hline Comprehension, \% ( $n)$ & $25(n=4)$ & $48(n=12)$ & 2.2 & 0.14 \\
\hline Repetition, \% ( $n)$ & $38(n=6)$ & $64(n=16)$ & 2.8 & 0.09 \\
\hline Spontaneous language fluency, $\%(n)$ & $56(n=9)$ & $52(n=13)$ & 0.3 & 0.6 \\
\hline Naming, \% (n) & $81(n=13)$ & $88(n=22)$ & 0.4 & 0.55 \\
\hline
\end{tabular}

Table 3 Imaging features

Anterior frontal to the anterior commissure, posterior caudal to the posterior commissure

\begin{tabular}{lllll}
\hline Talairach location & RH & LH & $\chi^{2}$ & $P$ \\
\hline Mean number of impaired Talairach boxes & $21.56 \pm 18$ & $20.4 \pm 14$ & & \\
Anterior box, \% & 39 & 33 & 3.44 & 0.06 \\
Posterior box, \% & 32 & 39 & 4.54 & 0.03 \\
Rolandic box, \% & 29 & 28 & 0.09 & 0.76 \\
\hline
\end{tabular}

(Table 3). In the RHSA group, fewer posterior cuboids (caudal to the posterior commissure) were related to aphasia than in the LHSA group.

Lesions inducing nonfluent aphasia were distributed symmetrically in both hemispheres. We found more anterior lesions inducing a disorder of comprehension and repetition after RHSA than after LHSA (Fisher's exact test, $P<0.05$ ) (Fig. 1).

Observation of individual data showed that some lesions inducing comprehension disorders involved the inferior frontal gyrus after RHSA, whereas after LHSA we did not find lesions in this anterior location, but rather in the more posterior temporal gyrus. Lesions in the inferior frontal gyrus were associated with impairment of repetition only after RHSA, while the inferior parietal lobule was associated with repetition disorder exclusively after LHSA. Nonfluent aphasias were associated in both groups with lesions in the basal ganglia, insula, and inferior frontal gyrus, but with temporal lesions only in the RHSA group (see Table 4 for a precise anatomical description).

\section{Discussion}

The main findings of this study are the following: (1) comprehension and also repetition were less frequently impaired after RHSA than LHSA in the hyperacute phase only, (2) in RHSA, comprehension had recovered more frequently at the second evaluation in the non-right-handed patients, (3) there were nine (56\%) non-right-handed patients in the RHSA group and none in the LHSA group, (4) RHSA was associated with less posterior lesions, and (5) in RHSA, comprehension impairment was more often associated with anterior lesion.

Concerning aphasia difference between RHSA and LHSA, comprehension and also repetition on admission (hyperacute phase) were less frequently impaired in RHSA than in LHSA. These differences disappeared during the 2 weeks' follow-up. Relative preservation of comprehension had been already suggested in crossed aphasia literature $[12,20]$. We explained this finding, observed in our group only in the hyperacute phase, due to the presence of left-handed and ambidextrous patients.

Concerning repetition, two studies in LHSA [15, 28] had shown that improvement in repetition was more variable than in other language functions. Moreover, repetition is one of the most "localized" language modality: analyses of language impairment after stroke had demonstrated that repetition disorders were intimately linked with lesions of the left insula [18]. In our study, we found a difference for repetition between right and left hemispheres only in the hyperacute phase, which was consistent with other data suggesting early plasticity mechanism [4].

Since manual dominance was known to be a factor for atypical language dominance, we compared language performance of right-handers and non-right-handers in RHSA. Comprehension was preserved after the 3rd day only in the non-right-handed group. This finding suggested that, in the case of RHSA, comprehension was less lateralized in the right hemisphere if patients were left-handed 
Imaging correlation
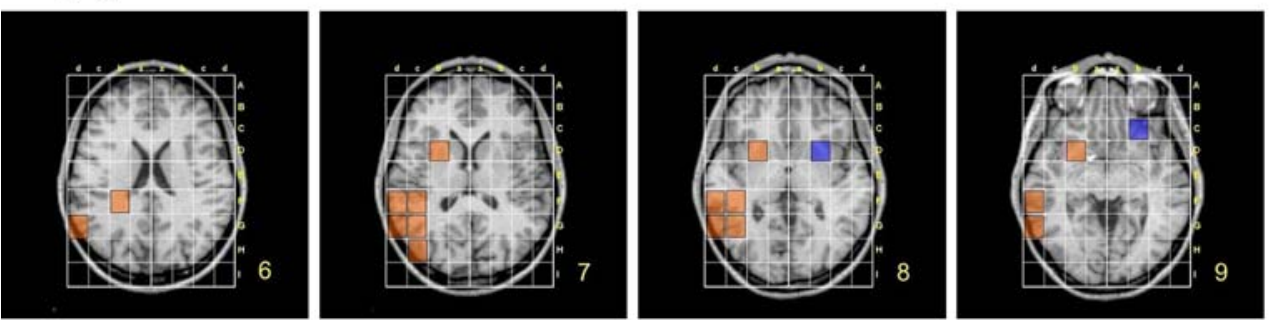

Comprehension impairment
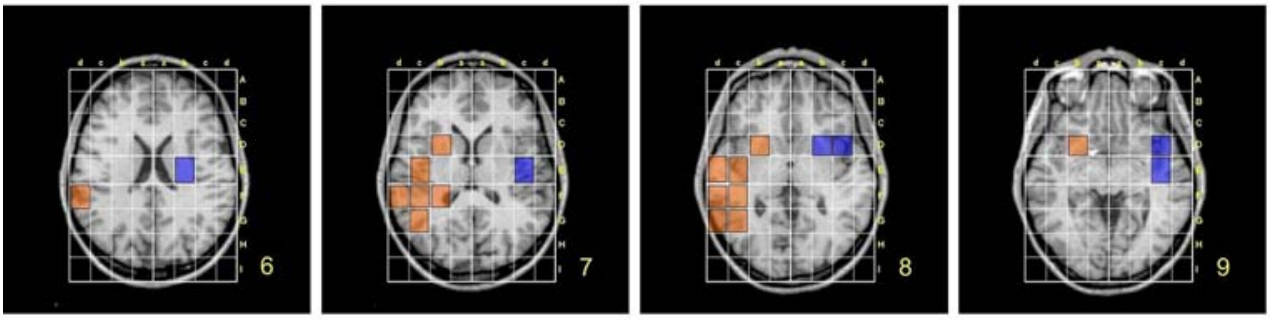

Repetition impairment
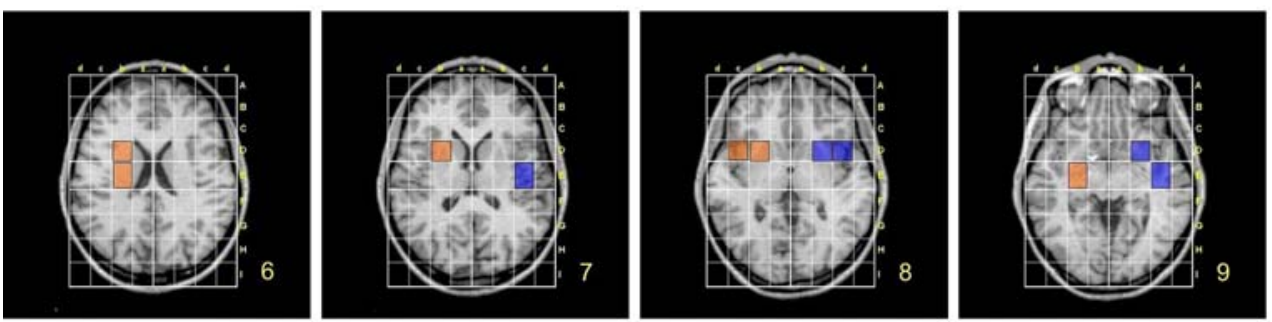

$\mathrm{RH}$ aphasia

LH aphasia

Fluency impairment

Fig. 1 Anatomo-clinical associations for three language modalities (repetition, comprehension, and spontaneous language fluency). Each colored box represents infarct locations significantly present (Fisher's exact test) in at least $50 \%$ of patients for each group (see "Methods" for details)

Table 4 Brain structures relevant to language modalities

\begin{tabular}{|c|c|c|}
\hline \multirow{2}{*}{$\begin{array}{l}\text { Impaired language } \\
\text { modality }\end{array}$} & \multicolumn{2}{|l|}{ Relevant structure } \\
\hline & RH & LH \\
\hline \multirow[t]{3}{*}{ Comprehension } & Putamen, claustrum, insula & Putamen, claustrum, insula, superior lateral fascicle \\
\hline & Frontal lobe: inferior gyrus & Temporal lobe: superior, middle, inferior, transverse gyrus \\
\hline & & Parietal lobe: inferior lobulus \\
\hline \multirow[t]{3}{*}{$\begin{array}{l}\text { Spontaneous language } \\
\text { fluency }\end{array}$} & Putamen, claustrum, insula & $\begin{array}{l}\text { Putamen, claustrum, insula, caudatus nucleus, superior lateral } \\
\text { fascicle, globus pallidus }\end{array}$ \\
\hline & Frontal lobe: inferior gyrus & Frontal lobe: inferior gyrus \\
\hline & Temporal lobe: superior and middle gyrus & \\
\hline \multirow[t]{4}{*}{ Repetition } & $\begin{array}{l}\text { Putamen, claustrum, insula, caudatus nucleus, } \\
\text { superior lateral fascicle }\end{array}$ & $\begin{array}{l}\text { Putamen, claustrum, insula, superior lateral fascicle, caudatus } \\
\text { nucleus }\end{array}$ \\
\hline & Frontal lobe: inferior frontal gyrus & \\
\hline & Temporal lobe: superior and middle gyrus & Temporal lobe: superior, middle, transverse gyrus \\
\hline & & Parietal lobe: inferior lobulus \\
\hline
\end{tabular}

or ambidextrous. The absence of left-handed patients with LHSA prevented us from performing the same analysis in this group. However, a lower frequency of comprehension impairment among left-handed aphasic patients, regardless of the side of the lesion - as already reported in an earlier study [9] - confirmed that comprehension and phonological processes in left-handers might be less severe than in right-handed patients. 
In the recent literature, the proportion of fluent versus nonfluent RHSA varies. Most of the studies showed that two-thirds of crossed aphasic patients were nonfluent, as after LHSA [5, 19]. However, the ratio found in our study contrasted with two recent reviews on crossed aphasia [20] and uncrossed aphasia [7], where the authors found only $50 \%$ of nonfluent aphasia. In our study the slightly higher ratio for both groups, with $56 \%$ nonfluent RHSA and $68 \%$ nonfluent LHSA, could be due to the early evaluation of aphasia, since patients might be more fluent in the postacute phase.

As expected, the percentage of left-handed and ambidextrous patients $(56 \%)$ was significantly greater in RHSA than in LHSA $(0 \%)$. This confirmed data from functional transcranial Doppler experiments, which showed in healthy subjects a flow increase in the corresponding area during a cognitive task. In such experiments, incidence of RH language dominance increased linearly with the degree of lefthandedness, from $4 \%$ in strongly right-handed to $27 \%$ in strongly left-handed people [17]. In another study using repetitive transcranial magnetic stimulation to induce speech arrest in 50 healthy volunteers, 57\% of ambidextrous people had bilateral language representation [16].

The motor and sensory motor dysfunctions present in all patients with RHSA versus only $72 \%$ of LHSA patients could suggest more extensive lesions inducing RHSA. However, imaging analysis showed that this was due to a more anterior location of lesion in RHSA. Spatial neglect, unlike anosognosia, was more related to $\mathrm{RH}$ than to $\mathrm{LH}$ lesion, suggesting that atypical language localization and space representation did not necessarily coexist [20].

Although we postulated previously that lesion inducing aphasia would be greater with RH than LH stroke, the imaging findings demonstrated that the size of lesions was similar after RHSA and LHSA with a comparable scale of aphasia. Topographic analysis of the lesions showed that the proportion of posterior lesions (caudal to the posterior commissure) was smaller after RHSA than after LHSA. When we compared the distribution of lesions inducing impairments of spontaneous language fluency, repetition or comprehension, we demonstrated that lesion-behavior relationship could be atypical after crossed aphasia. Indeed, we found that few right temporal lesions induced fluency disorder, in accordance with previous studies [1, 5, 10, 13]. In the same way, some right inferior frontal gyrus lesions induced comprehension and repetition impairment.

Our study is original because of an identical systematic evaluation in all patients and the presence of both patients with pure crossed aphasia and patients with atypical manual laterality. However, due to the rarity of aphasia after RHS in the general population, we performed a retrospective study with some limitations. The delay before the second assessment was not exactly the same for each patient but varied between 3 and 14 days in both groups. $\mathrm{CT}$ and MRI were also performed at different moments during the first 2 weeks after the stroke. Classically, brainbehavior association after stroke has been studied in more subacute-chronic phases ( 3 weeks- 3 months), when the lesion is stabilized and compensation is thought still to be minimal $[3,22]$. However, recent research suggests that the acute-stage evaluation can be of interest to capture a more specific brain-behavior relationship and to avoid misleading results caused by early brain plasticity [11]. For this reason, we associated first-day clinical data with imaging data from the following 2 weeks. This same interval for RHSA and LHSA anatomo-clinical associations allowed us to do a reliable comparison.

Moreover the consecutive group of LHSA did not include non-right-handed patients, which prevented us from comparing and interpreting the role of handedness in this population.

The aim of this study was to compare two groups with the most specific inclusion and exclusion criteria and the same quality of neurological and neuropsychological evaluations, which limited the size of the cohort due to the rarity of RHSA.

A multicentric prospective study, conducted during many years, including patients assessed exactly at the same time (clinical and imaging), with a control cohort of LHSA patients matched to the RHSA patients in terms of size and site of lesion, would be interesting to confirm the differences found in our study in aphasic language profile between the two groups.

\section{Conclusion}

Aphasics after RHSA, especially patients with atypical dominance, were less impaired in repetition and comprehension during the acute phase, suggesting probably a lesslateralized language area in this population.

RHSA lesions had the same average size according to the specific language disorder as LHSA lesions. However, in RHSA, we found lesions in the frontal gyrus in patients with comprehension and repetition impairment, and temporal lesions in patients with fluency impairment, suggesting atypical language organization after RHSA, particularly in non-right-handed patients in whom language is represented in the right hemisphere.

\section{References}

1. Alexander MP, Annett M (1996) Crossed aphasia and related anomalies of cerebral organization: case reports and a genetic hypothesis. Brain Lang 55(2):213-239 
2. Alexander MP, Fischette MR, Fischer RS (1989) Crossed aphasias can be mirror image or anomalous. Case reports, review and hypothesis. Brain 112:953-973

3. Basso A, Lecours AR, Moraschini S, Vanier M (1985) Anatomoclinical correlations of the aphasias as defined through computerized tomography: exception. Brain Lang 26:201-229

4. Bütefisch CM, Kleiser R, Seitz RJ (2006) Post-lesional cerebral reorganisation: evidence from functional neuroimaging and transcranial magnetic stimulation. J Physiol Paris 99(4-6):437-454

5. Carr MS, Jacobson T, Boller F (1981) Crossed aphasia: analysis of four cases. Brain Lang 14:190-202

6. Croquelois A, Wintermark M, Reichhart M, Meuli R, Bogousslavsky J (2003) Aphasia in hyperacute stroke: language follows brain penumbra dynamics. Ann Neurol 54:321-329

7. Godefroy O, Dubois C, Debachy B, Leclerc M, Kreisler A (2002) Lille stroke program.Vascular aphasias: main characteristics of patients hospitalized in acute stroke units. Stroke 33:702-705

8. Habib M, Joanette Y, Ali-Cherif A, Poncet M (1983) Crossed aphasia in dextrals: a case report with special reference to site of lesion. Neuropsychologia 21(4):413-418

9. Hecaen H, Ajuriaguerra J (1963) Les gauchers. Prévalence manuelle et dominance cérébrale. Presses Universitaires de France, Paris

10. Hécaen H, Mazars G, Ramier AM, Goldblum MC, Mérienne L (1971) L'aphasie croisée chez un sujet bilingue (vietnamienfrançais). Rev Neurol 124:319-323

11. Hillis AE, Newhart M, Heidler J, Barker PB, Herskovits EH, Degaonkar M (2005) Anatomy of spatial attention: insights from perfusion imaging and hemispatial neglect in acute stroke. $\mathrm{J}$ Neurosci 23:3161-3167

12. Joanette Y (1989) Aphasia in left-handers and crossed aphasia. In: Boller F, Grafman J (eds) Handbook of neuropsychology, vol 2. Elsevier, Amsterdam, pp 173-183

13. Joanette Y, Puel M, Nespoulous JL, Rascol A, Lecours AR (1982) Aphasie croisée chez les droitiers. Rev Neurol 138:575586

14. Jörgens S, Kleiser R, Indefrey P, Seitz RJ (2007) Handedness and functional MRI-activation patterns in sentence processing. Neuroreport 18(13):1339-1343
15. Kenin M, Swisher LP (1972) A study of pattern of recovery in aphasia. Cortex 8(1):56-68

16. Khedr EM, Hamed E, Said A, Basahi J (2002) Handedness and language cerebral lateralization. Eur J Appl Physiol 87:469-473

17. Knecht S, Drager B, Deppe M, Bobe L, Lohmann H, Flöel A, Ringelstein EB, Henningsen H (2000) Handedness and hemispheric language dominance in healthy humans. Brain 123:25122518

18. Kreisler A, Godefroy O, Delmaire C, Debachy B, Leclercq M, Pruvo JP, Leys D (2000) The anatomy of aphasia revisited. Neurology 54(5):1117-1123

19. Marien P, Engelborghs S, Vignolo LA, De Deyn PP (2001) The many faces of crossed aphasia in dextrals: report of nine cases and review of the literature. Eur J Neurol 8:643-658

20. Marien P, Paghera B, De Deyn PP, Vignolo LA (2004) Adult crossed aphasia in dextrals revisited. Cortex 40:41-74

21. Mazaux JM, Orgogozo JM (1982) Echelle d'évaluation de l'aphasie adaptée du Boston diagnostic aphasia examination. EAP Editions Psychotechniques, Paris

22. Mazzocchi F, Vignolo LA (1979) Localisation of lesions in aphasia: clinical-CT scan correlations in stroke patients. Cortex 15:627-654

23. Nespoulous JL, Lecours AR, Lafond D, Joanette Y, Lemay A, Puel M, Cot F, Rascol A (1992) Protocole Montréal-Toulouse d'examen linguistique de l'aphasie, MT 86, Module standard initial. Ortho Editions, Montréal

24. Oldfiedl RC (1971) The assessment and analysis of handedness: the Edinburgh inventory. Neuropsychologia 9:97-113

25. Pedersen PM, Vinter K, Olsen TS (2004) Aphasia after stroke: type, severity and prognosis. The Copenhagen aphasia study. Cerebrovasc Dis 17:35-43

26. Stone SP, Wilson B, Wroot A, Halligan PW, Lange LS, Marshall JC, Greenwood RJ (1991) The assessment of visuo-spatial neglect after acute stroke. J Neurol Neurosurg Psychiatry 54:345350

27. Talairach J, Tournoux P (1988) Co-planar stereotaxic atlas of the human brain. Thieme, New York

28. Vignolo LA (1964) Evolution of aphasia and language rehabilitation: a retrospective exploratory study. Cortex 1:344-361 